\title{
Expulsive choroidal haemorrhage: an experimental study
}

\author{
H. ZAUBERMAN \\ From the Department of Ophthalmology, Hadassah University Hospital, Jerusalem, \\ Israel
}

SUMmaRY The closure of 2 or more vortex veins in rabbits' eyes results in a persistent rise in intraocular pressure. In these circumstances a small opening into the anterior chamber at the corneoscleral limbus resulted in an iris prolapse, while a larger limbal wound was immediately followed by a massive choroidal expulsive haemorrhage. It appears from this experimental study that a definite and persistent rise in intraocular pressure could be a sign of impaired venous outflow and that the occurrence of an iris prolapse after a small limbal opening into the anterior chamber should prompt us to examine the fundus of the eye and look for a choroidal complication. It is possible that the present set of experiments is relevant to the mechanism of expulsive haemorrhage in man.

Expulsive choroidal haemorrhage is a rare but dreaded complication of intraocular surgery. It appears that patients suffering from glaucoma, hypertension, diabetes, and high myopia have a higher chance of having this complication than normal patients.

The histopathology of human eyes removed after an expulsive choroidal haemorrhage has been described by Manschot. ${ }^{1} \mathrm{He}$ reported 6 cases which prior to surgery suffered from glaucoma. Five of the six cases showed a localised necrosis of one or more ciliary arteries. He postulated that an increase in the intraocular pressure causes arteriolar collapse and accelerates degeneration of the vessel wall, thus predisposing it to a rupture.

In one case ${ }^{2}$ of spontaneous expulsive haemorrhage after a spontaneous rupture of a corneal ulcer the posterior ciliary artery appeared torn as it entered the suprachoroidal space.

From these reports it appears that a failure in the arterial circulation plus the sudden decompression of the eye, especially if it is glaucomatous, are the prime factors producing a massive choroidal haemorrhage. In the present experimental study I propose to investigate whether a failure in the venous choroidal outflow could be a factor in the development of an expulsive choroidal haemorrhage.

Correspondence to Professor H. Zauberman, Department of Ophthalmology, Hadassah University Hospital, PO Box 12000 , 91120 Jerusalem, Israel.

\section{Materials and methods}

Twelve albino rabbits were used in this study. The animals were anaesthetised with intravenous pentobarbitol $20 \mathrm{mg} / \mathrm{kg}$ and a $0.25 \mathrm{ml}$ retrobulbar injection of Novocain (procaine) $2 \%$.

In each rabbit the right eye was treated as follows: the conjunctiva was sectioned above the insertion of the lateral rectus muscle in 6 rabbits and above both insertions of the lateral and the internal recti muscles in the other 6 . The sclera below the muscles was exposed by displacing the muscle fibres with a muscle hook. In 6 rabbits the 2 vortex veins underneath the upper and lower margins of this muscle were closed tightly with the aid of a $7 / 0$ silk suture. In 3 rabbits 3 vortex veins were closed, 2 under the lateral rectus and one under the internal rectus muscle. In 3 rabbits 4 vortex veins were closed.

In every experiment the area of iris previously drained by the closed vortex vein was seen to become congested. When 2 or more vortex veins were occluded, petechiae appeared on the iris surface, and the choroid became congested in the affected area. In the rabbit the vortex veins drain not only the choroidal circulation but also the anterior segment of the eye, and this is why petechiae appear over the iris tissue when 2 or more vortex veins are occluded.

When 4 vortex veins were occluded, a mild haemorrhage was seen to appear on the iris near the angle. The choroid was seen to become quite congested, but no bleeding was detected in this tissue. 


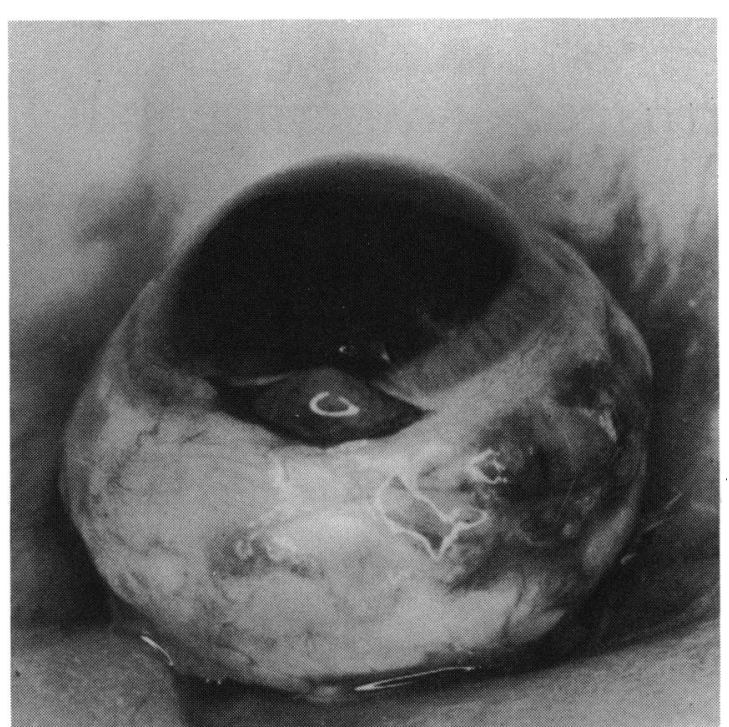

Fig. 1 Small iris prolapse is seen after limbal opening into the anterior chamber.

Prior to the retrobulbar injection of procaine and 5 minutes after the ligature of the veins the intraocular pressure was measured with the help of a Schiфtz tonometer in each of the 24 eyes under study.

Ten to 15 minutes after the closure of the veins the anterior chamber was entered through the corneoscleral limbus with the help of a sharp razor blade.

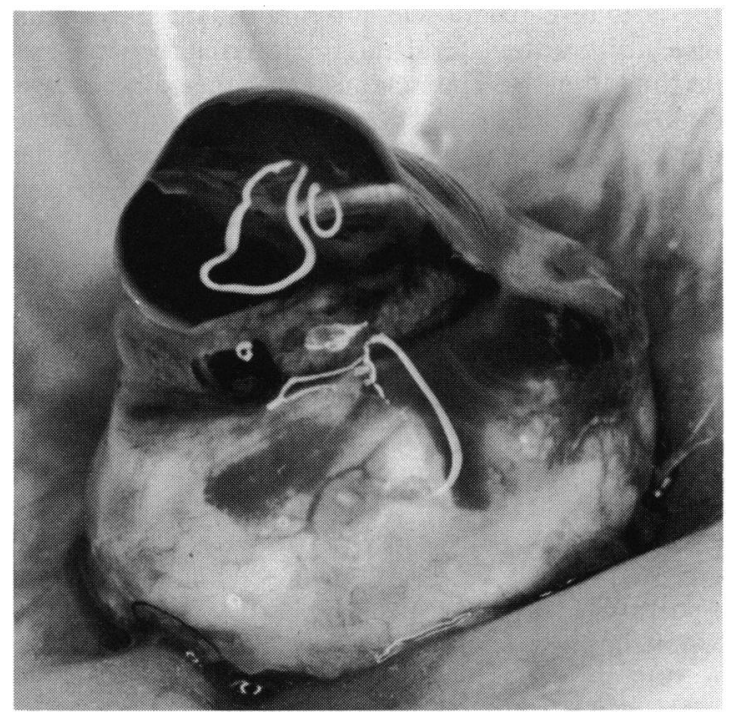

Fig. 2 Expulsion of lens and vitreous resulting from choroidal haemorrhage.
Then the corneal wound was enlarged along the limbus for a total length of $12 \mathrm{~mm}$.

For the purposes of control the left eyes of the 12 rabbits underwent retrobulbar anaesthesia and measurement of the intraocular pressure before and after it. In each case a corneoscleral penetrating wound of $12 \mathrm{~mm}$ was induced as in the right eye.

\section{Results}

In the 12 eyes without vortex vein occlusion the intraocular pressure was of $16-18 \mathrm{mmHg}$ before and after the retrobulbar injection of procaine. In the 6 eyes with occlusion of 2 vortex veins the intraocular pressure rose from $16-18 \mathrm{mmHg}$ before the vein ligature to $22-24 \mathrm{mmHg} 5$ minutes after the ligature. In 2 of these 6 eyes the $3 \mathrm{~mm}$ limbal opening of the anterior chamber produced by the razor blade resulted in an instantaneous iris prolapse (Fig. 1), which could not be replaced by repeated trials. When this incision was enlarged, the whole upper iris prolapsed out of the eye. This was immediately followed by the spontaneous expulsion of the lens, a large amount of vitreous loss, and the development of a massive vitreous haemorrhage (Fig. 2). In the other 4 eyes with 2 vortex vein occlusions the corneal opening resulted in a moderate hyphaema but no other complications.

In the 6 eyes which underwent 3 vortex vein ligatures and in the 6 eyes which underwent 4 vein ligatures the intraocular pressure rose from 16-18 $\mathrm{mmHg}$ to $30-35 \mathrm{mmHg}$. In 3 eyes with 3 vein occlusions and in 5 with 4 vein occlusions an expulsive haemorrhage occurred, as described above.

In the 24 control fellow eyes the corneoscleral

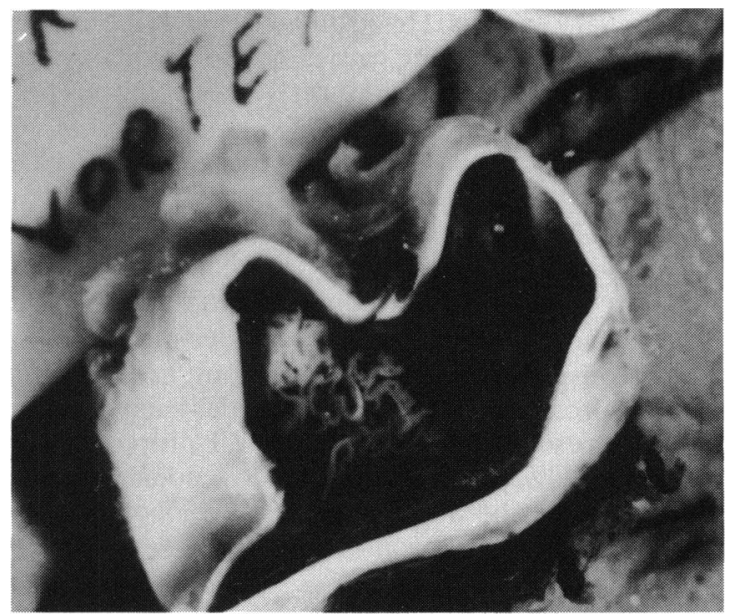

Fig 3 Gross section of rabbit's eye: ciliary body and choroidal haemorrhagic detachment and retinal detachment. 


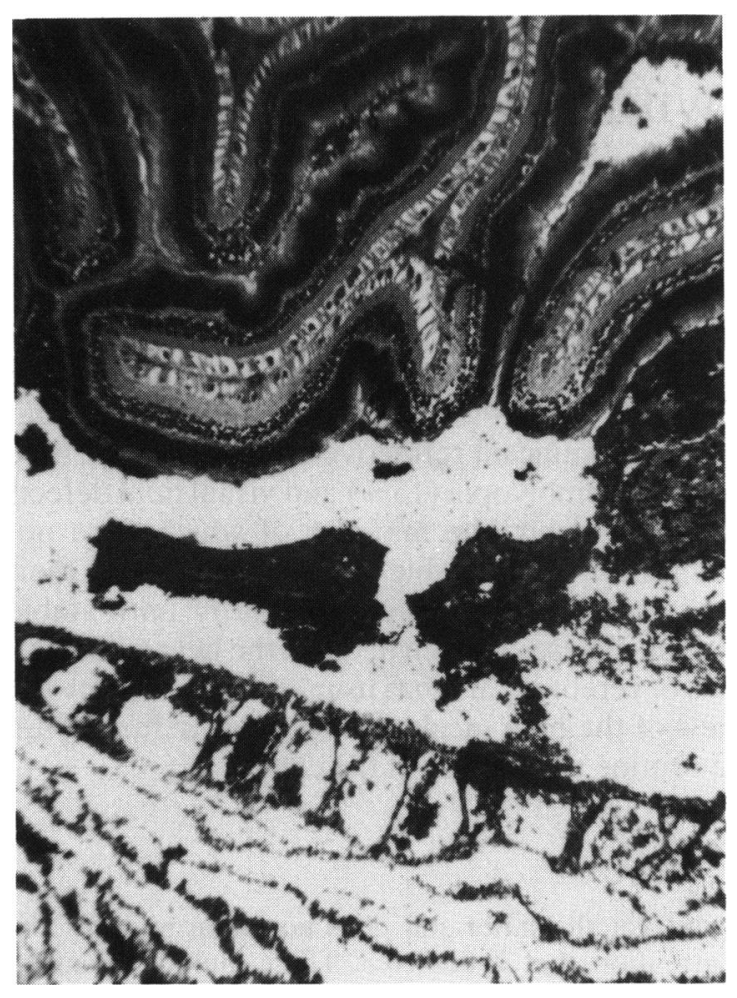

Fig. 4 Perichoroidal haemorrhage. Retinal detachment. $(\times 10)$.

opening into the anterior chamber was not followed by an iris prolapse or by a haemorrhage.

One eye affected with expulsive choroidal haemorrhage was enucleated and fixed in $10 \%$ formalin. Macroscopically this eye contained a haemorrhagic mass. The choroid and ciliary body were detached and the retina was detached and folded (Fig. 3).

Haematoxylin-eosin stained sections showed a severe choroidal and perichoroidal haemorrhage with a retinal detachment (Fig. 4) and a haemorrhage in the ciliary body.

\section{Discussion}

It appears from this study that it is possible to produce an experimental expulsive haemorrhage of the choroid by occluding partially or totally the venous outflow of an otherwise normal rabbit's eye.

In a previous study eyes removed after an expulsive choroidal haemorrhage were found to show necrotic changes in one or more posterior ciliary arteries. The rupture of the artery causing the haemorrhagic event was believed to be facilitated by the presence of previous degenerative changes in the vessel wall induced by an increased intraocular pressure, hypertension, diabetes, etc. ${ }^{1}$ The possibility that a rupture in a degenerated artery was secondary to stasis in the choroidal venous flow was not then considered. However, Samuels ${ }^{3}$ postulated that the invasion of fluid and blood into the perichoroidal space could result in traction on the arterial vessels and their rupture, and, if the bleeding is massive, the choroid may be ruptured, and the blood while flowing out of the globe carries along the intraocular contents. It is of interest that a blockage of venous outflow as demonstrated in the present study can reproduce so well the events of an expulsive choroidal haemorrhage. It is possible that a blockage in the venous outflow occurs occasionally during a surgical procedure in human eyes. A possible mechanism of venous closure could be pressure by the orbital contents on the vortex veins after an excessive amount of retrobulbar anaesthesia. Also, the accidental production of a retrobulbar haemorrhage could result in a compression of the vortex veins. Probably in those eyes in which the blood flow is already partially compromised, such as occurs in chronic glaucoma, systemic hypertension, and severe arteriosclerosis, the blockage of venous outflow is easier to obtain.

In the present set of experiments one important sign of venous flow occlusion has been a moderate to severe rise in intraocular pressure. This sign of venous stasis appears to be of importance. Indeed, a rise of the intraocular pressure as compared with the pressure prior to a retrobulbar injection could be a warning sign of venous outflow blockage. Furthermore, the presence of a spontaneous and recurrent iris prolapse after a small opening of the anterior chamber, such as occurred in this study, should make us aware of the possibility of venous stasis and lead us to examine the fundus of the eye for evidence of choroidal bleeding.

This study was supported in part by the Ignazius Sommers research fund.

\section{References}

1 Manschot WA. The pathology of expulsive hemorrhage. Am J Ophthalmol 1955; 40: 15-24.

2 Winslow RL, Stevenson W, Yanoff M. Spontaneous expulsive choroidal hemorrhage. Acta Ophthalmol (Kbh) 1974; 92: 33-6.

3 Samuels B. Postoperative non-expulsive subchoroidal hemorrhage. Arch Ophthalmol 1931; 6: 840-51. 